\title{
Enhancement of the Spitzer Infrared Array Camera Distortion Correction for Parallax Measurements
}

\author{
Patrick J. Lowrance ${ }^{\mathrm{a}}$, Sean J. Carey ${ }^{\mathrm{a}}$, James G. Ingalls ${ }^{\mathrm{a}}$, Jason A. Surace ${ }^{\mathrm{a}}$, \\ Peter Capak ${ }^{\mathrm{a}}$, John Stauffer ${ }^{\mathrm{a}}$, Chas Beichman ${ }^{\mathrm{b}}$, David Shupe ${ }^{\mathrm{b}}$, J. Davy Kirkpatrick ${ }^{\mathrm{b}}$ \\ ${ }^{a}$ Spitzer Science Center, 1200 East California Blvd, Pasadena, CA, USA 91125; \\ ${ }^{\mathrm{b}}$ Infrared Processing and Analysis Center, 770 S. Wilson Ave, Pasadena, CA 91125
}

\begin{abstract}
The Spitzer Space Telescope Infrared Array (IRAC) offers a rare opportunity to measure distances and determine physical properties of the faintest and coldest brown dwarfs. The current distortion correction is a $3^{\text {rd }}$ order polynomial represented by TAN-SIP parameters within the headers. The current correction, good to 100 mas, was derived from deep imaging, using marginally resolved galaxies in some cases, and has remained stable throughout both the cryogenic and warm mission. Using recent Spitzer calibration observations mapped to HST/ACS calibration observations of 47 Tuc with an absolute accuracy good to 1 mas, we are working towards a possible $5^{\text {th }}$ order polynomial correction that theoretically could allow measurements to within 20 mas. Extensive testing, using observations of 47 Tuc, NGC 6791 and NGC 2264, are underway, after which the new parameters will be used to update all the 3.6 and 4.5 um data taken within warm and cryogenic missions. We anticipate if achievable, this new accuracy could be combined with other ongoing enhancements (Ingalls et al, 9143-52) that will permit measurements of parallaxes out to about $50 \mathrm{pc}$, increasing the volume surveyed by a factor of 100, and enabling new capabilities such as luminosity measurements of the population of young brown dwarfs in the beta Pictoris moving group.
\end{abstract}

Keywords: infrared, distortion, calibrations, parallax, brown dwarfs

\section{INTRODUCTION}

The two InSb arrays of Infrared Array Camera ${ }^{1,2}$ (IRAC) aboard Spitzer Space Telescope ${ }^{3}$ are currently operating successfully into the fifth year past the original $\sim 5.5$ year cryogenic mission. Prior to the end of the cryogenic mission, it was noted that the passive cooling would continue to achieve telescope and multiple instrument chamber (MIC) temperatures of $\sim 30 \mathrm{~K}$. The thermal background of the telescope and the noise properties ${ }^{4}$ of the two InSb arrays of IRAC for passively cooled Spitzer provide comparable sensitivity ${ }^{5}$ to the cryogenic observations at 3.6 and $4.5 \mu \mathrm{m}$ during the post-cryogenic mission, i.e. the warm mission.

Images taken with the IRAC are distorted by a few percent relative to the sky grid. Early in the cryogenic mission, it was found these distortions could be well represented by polynomials, and a convention ${ }^{6}$ was developed for inserting the coefficients into the FITS header of each Basic Calibrated Data (BCD) product. The post-BCD software MOPEX accounts for these in co-addition of the images. Indeed, most software that is used currently for FITS images can use this convention and derive intermediate world coordinates from the pixel positions.

During the warm mission calibration, many tests were performed to check the distortion correction. The derived distortion correction was found to be unchanged, and therefore is the same for the entire mission. There current third order polynomial is currently good to $\sim 100$ mas, exceeding the mission requirements. In this paper, we present efforts being made to improve on this correction to increase the science potential.

\section{THE CURRENT CONVENTION}

The Simple Imaging Polynomial (SIP) convention was developed early in the cryogenic mission to provide a means for representing a non-linear geometric distortion with a polynomial within the FITS header keywords. As defined, $u, v$ are relative pixel coordinates with origin at CRPIX1, CRPIX2 and $x, y$ are "intermediate world coordinates" in degrees, with origin at CRVAL1, CRVAL2. Let $f(u, v)$ and $g(u, v)$ be the quadratic and higher- order terms of the distortion polynomial.

Space Telescopes and Instrumentation 2014: Optical, Infrared, and Millimeter Wave, edited by Jacobus M. Oschmann, Jr., Mark Clampin, Giovanni G. Fazio, Howard A. MacEwen, Proc. of SPIE Vol. 9143, 914358 - ( 2014 SPIE CCC code: $0277-786 \mathrm{X} / 14 / \$ 18 \cdot$ doi: $10.1117 / 12.2057195$ 
Then

$$
\left(\frac{x}{y}\right)=\left(\frac{C D 1 \_1}{C D 2 \_1} \frac{C D 1 \_2}{C D 2 \_2}\right)\left(\frac{u+f(u, v)}{v+g(u, v)}\right)
$$

We define $\mathrm{A} \_p \_q$ and $\mathrm{B} \_p \_q$ as the polynomial coefficients for polynomial terms $u^{p} v^{q}$. Then

$$
\begin{array}{ll}
f(u, v)=\sum_{p, q} A_{-} p_{-} q u^{p} v^{q}, & p+q \leq A_{-} \text {ORDER }, \\
g(u, v)=\sum_{p, q} B_{-} p_{-} q u^{p} v^{q}, & p+q \leq B_{-} \text {ORDER }
\end{array}
$$

Currently, the distortion is represented by a third order polynomial. Using the equations above, it can be written as

$$
f(u, v)=A_{-} 2 \_0 u^{2}+A_{-} 0 \_2 v^{2}+A_{-} 1 \_1 u v+A_{-} 1 \_2 u v^{2}+A_{-} 2 \_1 u^{2} v+A_{-} 3 \_0 u^{3}+A_{-} 0 \_3 v^{3}
$$

where the coefficients are listed within the header of the FITS images. The CDijj keywords encode skew as well as rotation and scaling. The $\mathrm{CD}$ - matrix values together with the higher-order distortion polynomials define a unique transformation from pixel coordinates to the plane-of-projection. For Spitzer, we also provide polynomials for the reverse transformation, for fast inversion.
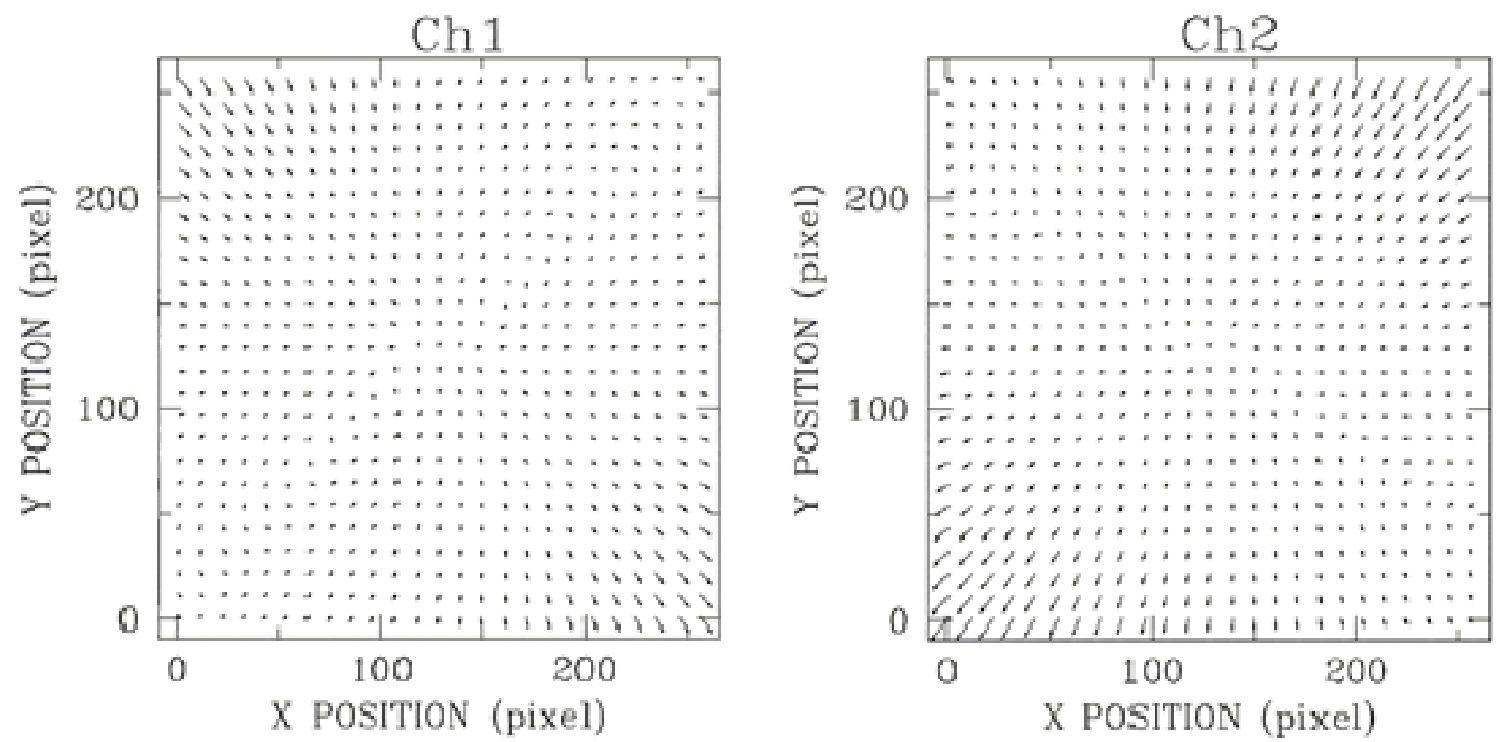

Figure 1: Optical image distortion in IRAC channels. The panels show the image distortions as calculated from a third order polynomial model that has been fit to in-flight data. The magnitude of the distortion and the direction to which objects have moved from their ideal tangential plane projected positions is shown with arrows. The length of the arrows has been increased by a factor of ten for clarity. 


\section{NEED FOR IMPROVED DISTORTION}

The current third order polynomial distortion correction per observation leaves a residual error of $\sim 100$ mas , or $<0.1$ pixel (Figure 1). This exceeds the needs for most science observations performed with IRAC. While there are other science drivers for improving the distortion, the study of nearby stars and brown dwarfs has been a main focus.

Brown dwarfs, or "failed stars" are objects that do not contain enough mass to turn on hydrogen fusion in the center. They are the lower limit of the star formation mass distribution, and the study of brown dwarfs in the solar neighborhood enables an unprecedented detailed look at the smallest masses possible. Recent all-sky surveys have revealed a previously unknown population and Spitzer can provide a new window on the nearest neighbors to the Sun.

An accurate distance measurement of the parallax of these objects is needed to fully analyze the apparent luminosity and relate it to the mass function. The average distance of $20 \mathrm{pc}$ requires a noise floor $<15 \%$ of $50 \mathrm{mas}$, or 7.5 mas. These objects are exceedingly dim to target with most ground based observatories and the fainter ones would take many years of dedicated time on larger 8 - to 10-m class telescopes. Spitzer makes it possible to characterize these objects because it observes in the wavelengths where they are brightest, and the telescope is free from atmospheric issues and thermal issues that affect other platforms. A program on an 8-m telescope would require roughly an order of magnitude more onsource time than Spitzer observations for the faintest objects.

We are currently working to achieve the desired correction within the boundaries of the current polynomial correction. The current approach was to increase the order of the polynomial correction from a third order to a fifth order. Expectations predicted an reduction in the noise floor by $\sim 5 \mathrm{x}$, or $\sim 20$ mas. From there the number of iterations of the observations could easily approach the 5-10mas needed for pushing the parallax measurable out to possibly 50pc. We have approached this problem using both dense stellar clusters and with a wide survey covering 2 square degrees.

\section{DERIVING THE NEW DISTORTION}

\subsection{Method 1: using dense clusters}

Using known globular clusters is a customary method for deriving a distortion correction. Given that these fields tend to be crowded, confusion in Spitzer imaging could create problems with centroiding for many fields. Therefore, a field 6' west of 47 Tuc, which has been used repeatedly by HST for calibration and monitoring of ACS performance, was identified. While this is a rich stellar field, it is far enough from the core of 47 Tuc that confusion was not a problem, so it was observed in the spring of 2013 with approximately 10,000 combined Spitzer/HST sightings (300-600 objects $\times 25$ sightings) with which to probe the Spitzer focal plane (Figure 2).

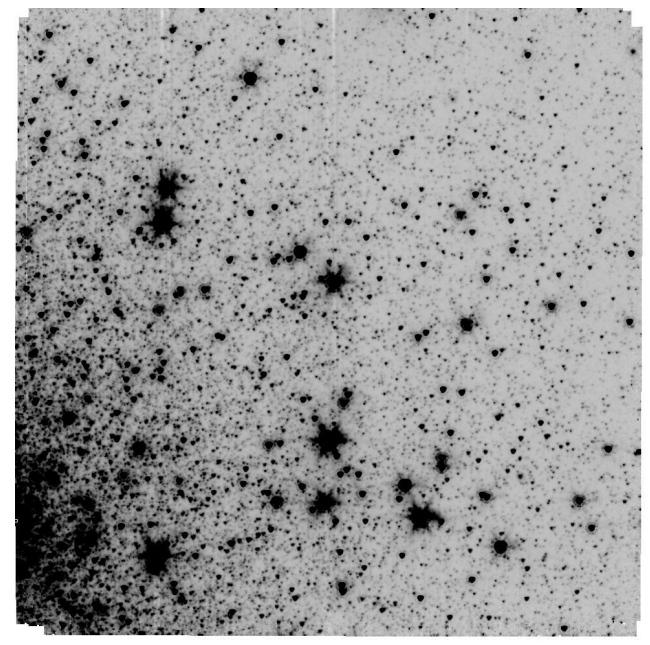

Figure 2: Ch2 mosaic of a field 6 arcmin W of 47 Tuc. We used HST ACS calibration observations of field (good to $\sim 1 \mathrm{mas)} \mathrm{to}$ determine a "best fit" distortion solution. We also used observation of NGC 6791 and NGC 2420 to test the solution. 
From the HST/ACS catalog approximately 2,000 sources with a flux at I brighter than 20 mag and separated by 2 arcsec were used. Each BCD frame was first adjusted to the HST frame by the use of the sources in the central 100 pixels. The typical offset was $<250$ mas. The frames that did not have sufficient overlap of HST sources were rejected as well. A match with the current $3^{\text {rd }}$ order frames found 9516 observations of sources.
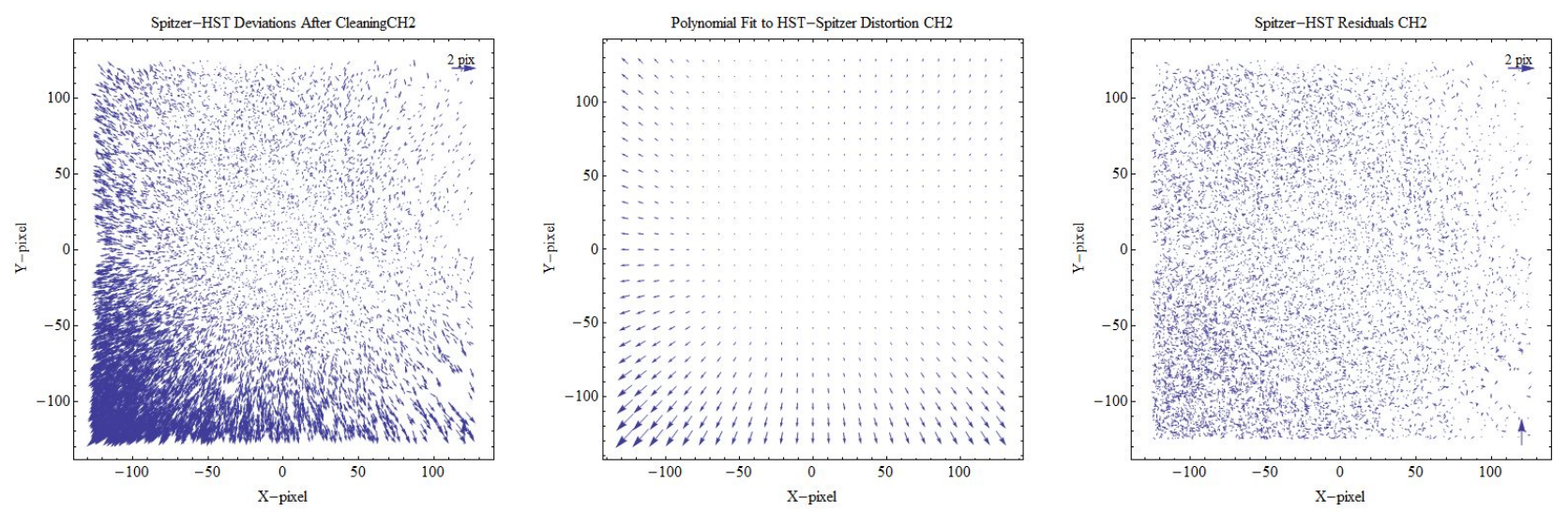

Figure 3: Ch2 mosaic of a field 6 arcmin W of 47 Tuc. We used HST ACS calibration observations of field (good to $\sim 1 \mathrm{mas)} \mathrm{to}$ determine a "best fit" distortion solution. We also used observation of NGC 6791 and NGC 2420 to test the solution.

The non-linear terms of the polynomial distortion correction were removed and a new solution was derived using a best fit to all the sources. Several passes were fit to the model, and objects rejected if found to be an outlier, defined as having vector amplitudes differing $>2$ sigma from the local average or theta $>60$ deg from nominal vector orientation at that position. This usually resulted in a $5-10 \%$ reduction in the number of sources. A new $5^{\text {th }}$ order polynomial was then fit to the data.

As can be seen in figure 3, the worst fit is near the bottom corner that is expected due to the crowding near the globular cluster. The least number of sources were retrievable near that corner compared to the full array. Residuals of the fit were calculated to be approximately similar to the $3^{\text {rd }}$ order. Several tests were run using different clusters as well comparing the new positions derived from the 5 th order to the positions from the $3^{\text {rd }}$ order.

To test the newly derived SIP polynomial coefficients, data sets were chosen from IRAC observations clusters NGC 6791 and NGC2420. Star centroids were calculated in each BCD and converted to RA/Dec using only the linear terms of the distortion contained in the CD matrix, while ignoring higher-order terms. Then a least squares fitting was performed to fit various models, including the currently used Polynomial_SIP, and Polynomial2d, Chebyshev2d, and Legendre2d, without weighting. The difference between SIP Polynomial and Polynomial2d is that the SIP_Polynomial allows the combination of the exponents to equal the order and Polynomial2d allows the highest exponent to be the order. For example, a $3^{\text {rd }}$ order Polynominal2d allows $x^{1} y^{3}, x^{2} y^{3}$, etc while the SIP_Polynomial does not.

Data cleaning was excluded except for a couple of experiments. Training sets were used to check for model over-fitting. The differences between measured and "undistorted" positions using as data combined with 10-fold cross-validation, in which $90 \%$ of data is used as training and $10 \%$ as test, repeated 10 times. The Legendre $2 \mathrm{~d}$ and Chebyshev $2 \mathrm{~d}$ polynomials gave a very similar answer as the Polynomial2d, so were no longer considered.

The preliminary results indicate the current $3^{\text {rd }}$ order SIP polynomial gives the lowest error on the test set for the majority of the datasets analyzed individually. Higher-order polynomials $(>3)$ decrease the in-sample error but increase the out-of-sample (test set) error, which indicate over-fitting (figure 4). The differences in the current SIP polynomial near the edges merit further investigation. 

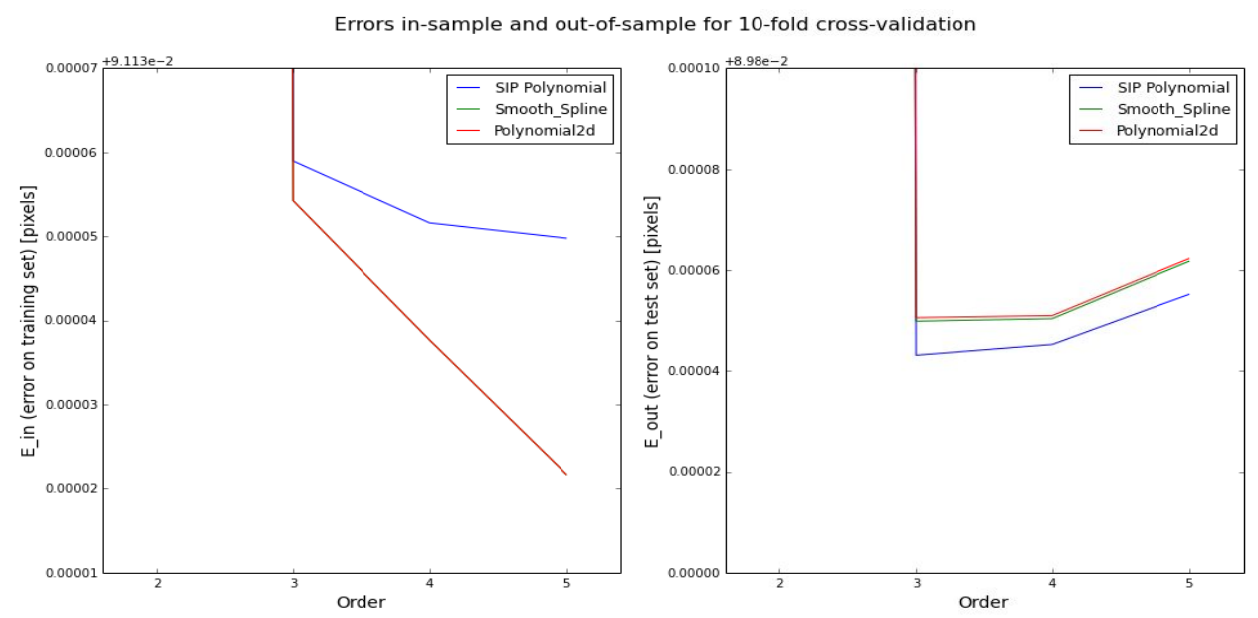

Figure 4: Errors found in the least squares fitting of both clusters (NGC 6791 and NGC 2420) data using a SIP Polynomial, Polynomial2d, and smooth Bivariate Spline without weighting or data. The Legendre $2 \mathrm{~d}$ and Chebyshev2d polynomials give the same answers as Polynomial2d so are not plotted. The orders higher than $3^{\text {rd }}$ appear to over fit the data. In-sample (i.e., training set) error decrease with high order, but out-of-sample (i.e. test set) error increase with higher order.

\subsection{Method 2: using large scale survey COSMOS}

The Cosmological Evolution Survey (COSMOS) ${ }^{8}$ is an astronomical survey designed to image a 2 square degree field with many of the major space-based telescopes (Hubble, Spitzer, GALEX, XMM, and Chandra) and many of the large ground based telescopes (VLA, CFHT, and UKIRT). The primary goal is to study the relationship between the largescale structure of the universe and the formation of galaxies. This survey contains over 75,000 IRAC exposures in each of IRAC Ch. 1 and 2, and has $<5$ mas absolute astrometry for $\sim 700,000$ sources. On average, there are $\sim 100$ isolated point sources per IRAC frame with known astrometry (Figure 5).

As an alternative to the globular clusters, the distortion parameters are also being derived using a chi-sq minimization technique commonly used for extragalactic science ${ }^{9}$. This method uses a grid of sources with known astrometric positions with multiple measurements of the relative positions of these sources with another instrument, in this case IRAC. Due to the large number of sources and frames, there are approximately 9 million measurements, or an average of 170 measures per pixel.
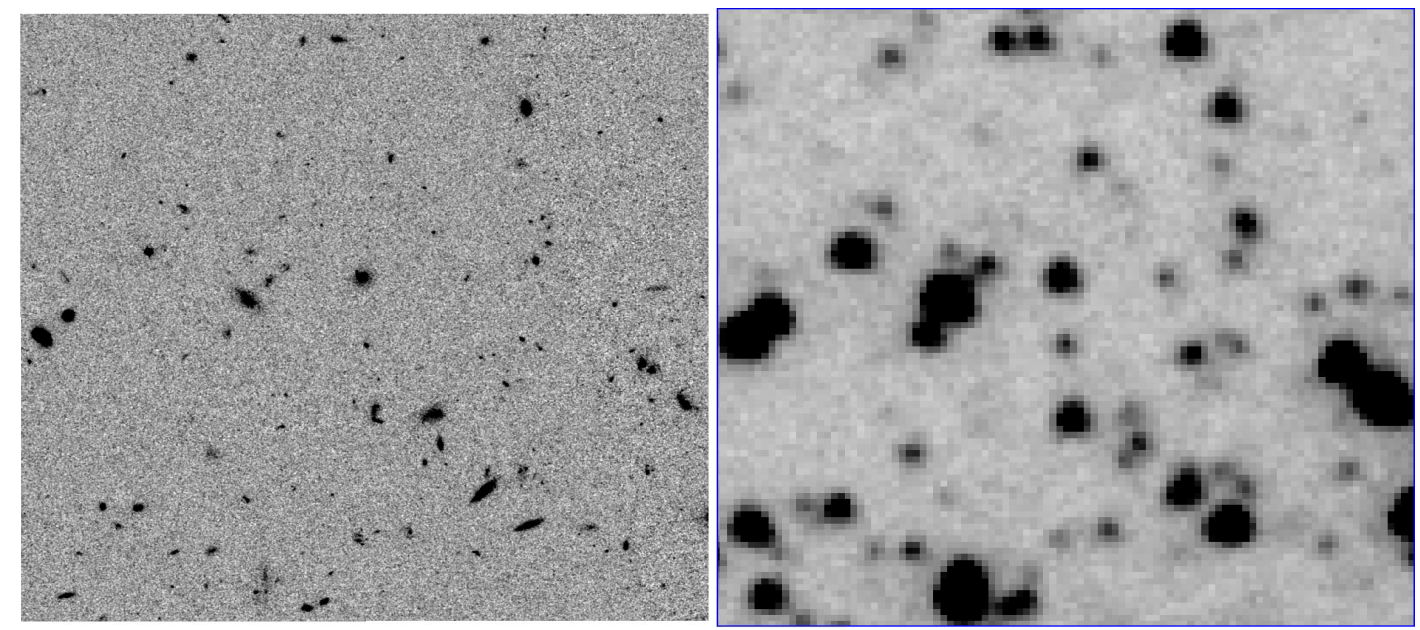

Figure 5: Small portion (1x1') of the COSMOS field (ACS on left and IRAC on right).

Preliminary results confirm the existing IRAC pixel scale and distortion map as well as recover the known offset of $\sim 0.2-0.5$ " between the 2MASS and true astrometric zero-point in this field. The current 3rd order polynomial was found 
to describe the distortion small residuals. There was a high-frequency component in the x-direction has an amplitude of approximately $+/-0.1$ pixels (Figure 6). This is still work in progress but it has been conjectured believe it comes from a small bias dependent on the time delay between frames. If so, this high frequency X component isn't going to be substantially by a higher order polynomial. Further tests are under way to determine the source and possibility of removal.

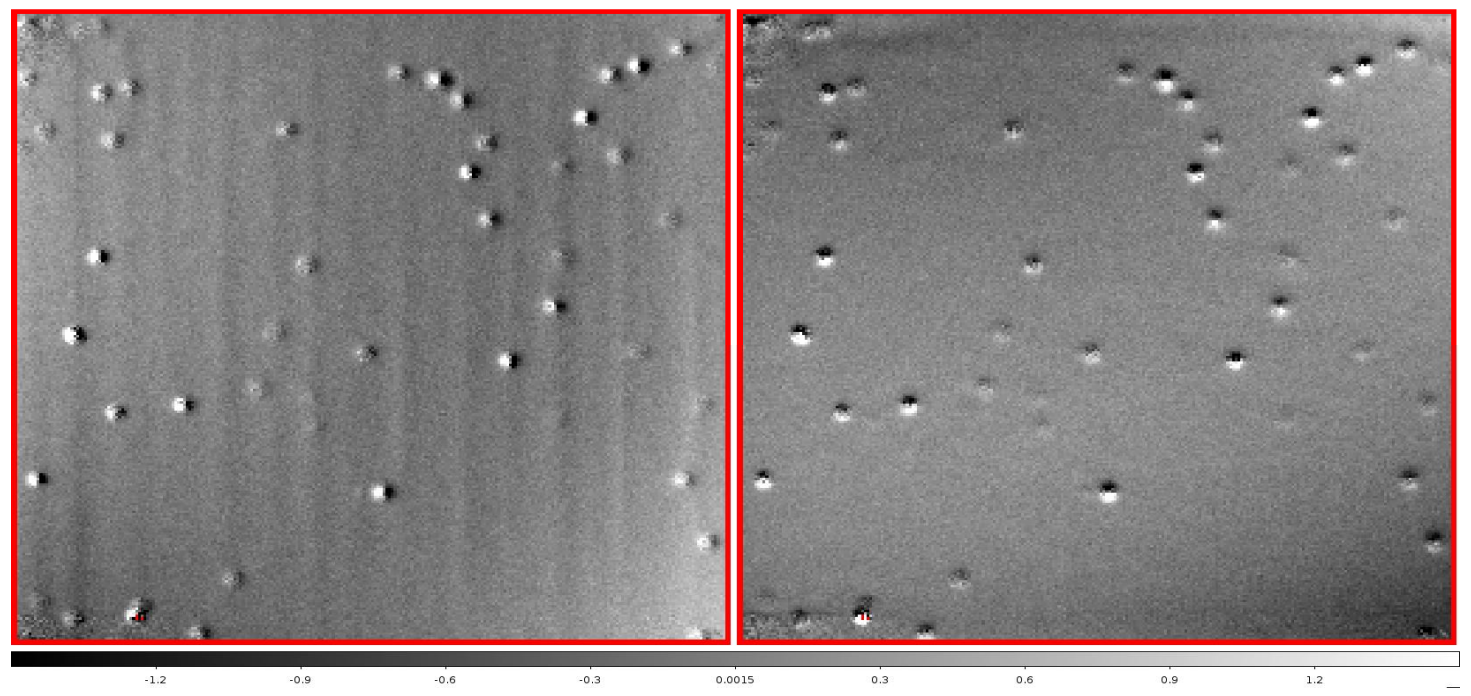

Figure 6: Residual distortion in IRAC Ch1 after removing the linear term in the $\mathrm{x}$ (left) and $\mathrm{y}$ (right) direction. The greyscale ranges from -1.5 to +1.5 pixels. The large spots are bad pixels that draw sources towards them. These can be removed. These maps are derived from 9.1 million measurements of sources with 5mas absolute astrometry in the COSMOS field.

\section{CONCLUSION}

The current third order polynomial distortion correction per observation leaves a residual error of $\sim 100 \mathrm{mas}$, or $<0.1$ pixel. This exceeds the needs for most science observations performed with IRAC. There now exists a rare opportunity to measure distances and determine physical properties of the faintest and coldest brown dwarfs and understand a significant amount about star formation and our nearest neighbors. An accurate distance measurement of the parallax of these objects is needed to fully analyze the data, but it requires a new distortion correction to bring the measurement error down near $\sim 20$ mas to derive the average parallaxes. If this is available, then combining a new distortion correction with the drift scan method discussed in Ingalls et al (9143-52) would be a new powerful tool in further expanding the available science.

Work is still in the preliminary stages. Two methods have been used to test the new derived distortion correction, including the globular cluster and large field survey. Both methods confirm the exiting IRAC pixel scale and distortion. The globular cluster least squares fitting suggest that residuals remaining after a $3^{\text {rd }}$ order fit were small because higher order polynomials appeared to over-fit the data. This could also be the limit of the data set being used.

Using the COSMOS large field observations, a second test was created that compared the astrometry of $\sim 9$ million objects with absolute astrometry good to 5mas in the HST/ACS observations. This test provided $\sim 180$ measures per pixel position in IRAC, and found the current $3^{\text {rd }}$ order polynomial to leave small residuals. It also revealed a high frequency residual in the $\mathrm{X}$ component of the distortion. We are attempting to fully understand this residual, but currently believe it comes from a small bias that is dependent on the time delay between frames being imprinted and not removed successfully in calibration frames. Once this is removed, a better fit might be obtained, but a polynomial might still not be the best method to correct the distortion to the level needed for the parallax measurements. Further work is still continuing. 


\section{REFERENCES}

[1] Fazio, G. G., Hora, J. L., Allen, L. E., Ashby, M. L. N., Barmby, P., Deutsch, L. K., Huang, J.-S., et al., "The Infrared Array Camera (IRAC) for the Spitzer Space Telescope", ApJS, 154, 10-17 (2004).

[2] Hora, J. L., Fazio, G. G., Allen, L. E., Ashby, M. L. N., Barmby, P., Deutsch, L. K., Huang, J.-S., et al., "In-flight performance and calibration of the Infrared Array Camera (IRAC) for the Spitzer Space Telescope", Proc. SPIE 5487, 77-92 (2004).

[3] Werner, M. N., Roellig, T. L., Low, F. J., Rieke, G. H., Rieke, M., Hoffmann, W. F., Young, E., et al., “The Spitzer Space Telescope Mission", ApJS, 154, 1-9 (2004).

[4] McMurtry, C. W., Pipher, J. L., Forrest, W. J., "Spitzer space telescope: dark current and total noise prediction for InSb detector arrays in the infrared array camera (IRAC) for the post-cryogen era", Proc. SPIE 6265, 08 (2006).

[5] Storrie-Lombardi, L. J., et al., "The Science Opportunities of the Warm Spitzer Mission”, AIP, 943 (2007).

[6] Shupe, D.L., Moshir, M., Li, J., Makovoz, D., Narron, R., Hook, R.N. "The SIP Convention for Representing Distortion in FITS Image Header", ADASS XIV ASP Conference Series, Vol. 347, Proceedings of the Conference held 24-27 October, 2004 in Pasadena, California, USA. Edited by P. Shopbell, M. Britton, and R. Ebert. San Francisco: Astronomical Society of the Pacific, p.491, (2005)

[7] Makovoz, D., \& Khan, I. 2004, in ASP Conf. Ser., Vol. 347, ADASS XIV, ed. P. L. Shop- bell, M. C. Britton, \& R. Ebert (San Francisco: ASP), 81

[8] Koekemoer, A.M., Aussel, H, Calzetti, D. et al "The COSMOS Survey: Hubble Space Telescope Advanced Camera for Surveys Observations and Data Processing” ApJS, 172, 196-202 (2007)

[9] Capak, P., Aussel, H., Ajiki, M., et al, "The First Release COSMOS Optical and Near-IR Data and Catalog”, ApJS 172, 99-116 (2007) 